\title{
Cuidado compartilhado entre profissionais de Centros de Atenção Psicossocial Adulto e Álcool e outras Drogas
}

\author{
Shared care between professionals from Adult and Alcohol and other Drugs Psychosocial \\ Care Centers

\begin{abstract}
Atención compartida entre profesionales de los Centros de Atención Psicosocial de Adultos y Alcohol y otras Drogas
\end{abstract}

Pérola Prado1, Gabriella de Andrade Boska², Priscilla de Oliveira Luz ${ }^{2 \star}$, Heloísa Garcia Claro ${ }^{3}$, Márcia Aparecida Ferreira de Oliveira², Cristhian Ricardo Schieck ${ }^{4}$.

\section{RESUMO}

Objetivo: Analisar a concepção dos profissionais acerca das ações de cuidado compartilhadas entre Centros de Atenção Psicossocial Adulto (CAPS Adulto) e Álcool e Drogas (CAPS AD). Métodos: Estudo qualitativo, interpretativo, realizado com 10 profissionais, seis de um CAPS Adulto e quatro de um CAPS AD de uma região de São Paulo. Aplicou-se questionário digital semiestruturado e utilizou-se a abordagem de análise temática do conteúdo. Os dados foram coletados em dezembro de 2018, atendendo todas as recomendações éticas. Resultados: Os dados foram organizados nas categorias temáticas: 1) o problema de saúde mental e o uso de álcool e outras drogas; 2) o Projeto Terapêutico Singular (PTS) de casos com duplo diagnóstico; 3) dificuldades e potencialidades no compartilhamento de casos entre CAPS Adulto e CAPS AD. Conclusão: Observou-se que o uso de álcool e outras drogas é negativamente associado aos problemas de saúde mental e que esta concepção interfere na organização do projeto terapêutico compartilhado entre os serviços. Além disso, a concepção dos profissionais é de que as ações compartilhadas entre os CAPS ampliam as possibilidades de cuidado, mas demandam trabalho em rede.

Palavras-chave: Saúde mental, Serviços de saúde mental, Transtornos relacionados ao uso de substâncias, Transtornos mentais, Comorbidade.

\begin{abstract}
Objective: To analyze the professionals' conception of the care actions shared between Adult and Alcohol and Drugs Psychosocial Care Centers (CAPS Adult and CAPS AD). Methods: This was a qualitative, interpretive study, carried out with 10 professionals, six from a CAPS Adult and four from a CAPS AD in a region of São Paulo. A semi-structured digital questionnaire was applied, and the thematic content analysis approach was used. Data were collected in December 2018, meeting all ethical recommendations. Results: The data were organized into thematic categories: 1) the mental health problem and the use of alcohol and other drugs; 2) the Single Therapeutic Project (PTS) of cases with dual diagnosis; 3) difficulties and potential in case sharing between CAPS Adult and CAPS AD. Conclusion: It was observed that the use of alcohol and other drugs is negatively associated with mental health problems and that this concept interferes in the organization of the therapeutic project shared between the services. In addition, the professionals' conception is that the actions shared between the two CAPS expand the possibilities of care, but demand networking.
\end{abstract}

Key words: Mental health, Mental health services, Substance use disorders, Mental disorders, Comorbidity.

1 Centro de Atenção Psicossocial Álcool e outras Drogas II, São Paulo - SP.

2 Universidade de São Paulo, São Paulo - SP. `E-mail: pris_luz@usp.br

3 Universidade Estadual de Campinas, Campinas - SP.

${ }^{4}$ Centro de Atenção Psicossocial Adulto III, São Paulo - SP. 


\section{RESUMEN}

Objetivo: Analizar la concepción de los profesionales sobre las acciones asistenciales compartidas entre los Centros de Atención Psicosocial de Adultos (CAPS Adultos) y Alcohol y Drogas (CAPS AD). Métodos: Estudio cualitativo, interpretativo, realizado con 10 profesionales, seis de un CAPS Adulto y cuatro de un CAPS AD en una región de São Paulo. Se aplicó un cuestionario digital semiestructurado y se utilizó el enfoque de análisis de contenido temático. Los datos se recopilaron en diciembre de 2018 , cumpliendo con todas las recomendaciones éticas. Resultados: Los datos se organizaron en categorías temáticas: 1) el problema de salud mental y el uso de alcohol y otras drogas; 2) o Proyecto Terapéutico Único (PTS) de casos con diagnóstico dual; 3) dificultades y potencial en compartición de casos entre CAPS Adult y CAPS AD. Conclusión: Se observó que el uso de alcohol y otras drogas se asocia negativamente con problemas de salud mental y que este concepto interfiere en la organización del proyecto terapéutico compartido entre los servicios. Además, la concepción de los profesionales es que las acciones compartidas entre los CAPS amplían las posibilidades de atención, pero exigen el trabajo en red.

Palabras clave: Salud mental, Servicios de salud mental, Trastornos relacionados con el uso de sustancias, Trastornos mentales, Comorbilidad.

\section{INTRODUÇÃO}

O atual modelo psicossocial de atenção às pessoas com problemas de saúde mental, incluindo as que sofrem por questões decorrentes do consumo de álcool e outras drogas é direcionado pela Lei da Reforma Psiquiátrica Brasileira, e reforçada pelos conceitos de Desinstitucionalização e Reabilitação Psicossocial (BRASIL, 2001). Essa proposta de cuidado é realizada preferencialmente por serviços de saúde mental comunitários e de base territorial, nomeados como Centros de Atenção Psicossocial Adulto (CAPS Adulto) e Álcool e Outras Drogas (CAPS AD), pertencentes à Rede de Atenção Psicossocial (RAPS) (BRASIL, 2011).

Esses serviços são pontos estratégicos da RAPS, pois estão próximos do convívio e das trocas sociais, afetivas e culturais dos usuários. De forma articulada, tem por objetivo propiciar a (re)inserção social e garantir o exercício pleno de cidadania das pessoas com necessidades de saúde mental, promovendo o acesso a cuidado de saúde integral e humanizado, a partir das demandas apresentadas pela população (DUARTE MV, et al., 2020).

Diante de suas especificidades, o CAPS Adulto e o CAPS AD, oferecem diversos recursos terapêuticos para o cuidado dos usuários: acompanhamento familiar, grupos, atendimentos individuais, atividades culturais, comunitárias, organizativas e de geração de trabalho e renda. Além disso, os CAPS do tipo III, tem funcionamento $24 \mathrm{~h}$ para acolhimento integral oferecendo maiores possibilidades de atenção e manejo de crises no próprio território, evitando internações psiquiátricas (LIMA DK e GUIMARÃES J, 2019; OLIVEIRA CA, et al., 2021).

No que se refere ao perfil dos usuários que acessam os serviços comunitários de saúde mental, estudos prévios relatam que a prevalência de comorbidades psiquiátricas, ou duplo diagnóstico, em pessoas com problemas do uso e abuso de álcool e outras drogas varia de $20 \%$ a $82 \%$ e para pessoas com diagnóstico de transtorno mental a prevalência do uso de álcool e outras drogas varia de $20 \%$ a $75 \%$ (BRASIL, 2002; TREVISAN ER, 2019). Quanto ao recurso do acolhimento integral, um estudo realizado em CAPS AD III mostrou que $33,2 \%$ das ocupações das camas são de usuários com comorbidades psiquiátricas (ARAÚJO AK e SOARES VL, 2018).

Esta associação é mais comumente observada em pessoas com diagnósticos de transtornos de humor, de pensamento e transtornos alimentares que geralmente fazem uso problemático de álcool, tabaco, opioides, maconha e cocaína (BOSKA GA et al., 2018; MESSER T et al., 2017; HUNT GE et al., 2018). Ainda se destaca que o uso dessas substâncias, mesmo em pequenas doses, aparece como dispositivo de amenização do sofrimento psíquico, mas que ao mesmo tempo pode gerar consequências e impactos que agravam o adoecimento e a vulnerabilidade, comprometendo a evolução do cuidado em saúde mental e por isso a 
necessidade de um tratamento que contemple o sujeito integralmente em ambas as condições (JONES CM e MACCANCE-KATZ EF, 2019).

Para além da presença ou não de comorbidades, análises fundamentais são feitas e apresentam o contexto sócio-histórico da saúde mental como um sistema imerso em crenças, culturas e que por muito tempo teve como principal base de conhecimento a vertente biomédica, de onde emergem os significados do sofrimento psíquico e o do uso de drogas. Um estudo que buscou descrever sobre a experiência da associação do consumo de álcool e outras drogas, como também sobre o sofrimento psíquico na perspectiva de usuários atendidos por CAPS, identificou que estes se descrevem de maneira fragmentada entre "doentes mentais" e "usuários de drogas" a partir do que os serviços, de forma excludente, reproduzem sobre esta identidade (FERNANDES MA, et al., 2018).

É necessário refletir sobre modelos de atenção abrangentes para essas pessoas que abordam o sofrimento psíquico associado ao uso de álcool e outras drogas, bem como, mais estudos informativos sobre ações práticas que contribuam para o aprimoramento deste cuidado (MESSER T, et al., 2017; HUNT GE, et al., 2018; FERNANDES MA, et al., 2018). Os CAPS correspondem a esse modelo de atenção, mas ainda é incipiente as produções científicas em relação às formas de cuidado e manejos necessários para usuários com duplo diagnóstico, a partir das experiências dos profissionais (VIEIRA FD, et al., 2017).

Com o intuito de contribuir com a discussão sobre a produção do cuidado para pessoas com diagnóstico de transtorno mental e que fazem uso de álcool e outras drogas, este estudo teve por objetivo analisar a concepção dos profissionais acerca das ações de cuidado compartilhadas entre CAPS Adulto e CAPS AD.

\section{MÉTODOS}

Pesquisa interpretativa de abordagem qualitativa, que permite a atribuição de sentido e intencionalidade às relações, opiniões e interpretações e busca compreender a complexidade do fenômeno das ações de cuidado compartilhadas em saúde mental (ROMANINI M, et al., 2017).

Dois CAPS do município de São Paulo foram cenário deste estudo: um CAPS Adulto III e um CAPS AD II, ambos serviços referência para o mesmo território. A coleta de dados foi realizada no mês de dezembro de $2018 \mathrm{com} 10$ profissionais dos serviços selecionados. Participaram seis profissionais do CAPS Adulto e quatro do CAPS AD, tanto de nível superior ou técnico que aceitaram participar da pesquisa.

Foram incluídos profissionais em trabalho ativo em um dos dois CAPS (não estar em período de licença ou férias) e foram excluídos aqueles que trabalhavam no período noturno e exclusivamente nas questões administrativas, por não terem contato direto com outros profissionais da rede para articulação das ações de cuidado.

Os participantes do estudo foram abordados e incluídos após uma participação em reunião geral de equipe, realizada em ambos os serviços, para apresentação do projeto, convite e coleta de endereços de emails dos profissionais interessados. Neste momento, foi assinado presencialmente o Termo de Consentimento Livre e Esclarecido (TCLE) por 28 profissionais dentre os 49 que atenderam ao critério de inclusão. Em seguida, o convite foi oficializado via correio eletrônico juntamente com o link para acesso ao questionário aberto.

Optou-se pelo formato de questionário eletrônico pela praticidade em meio à reestruturação de gestão e processo de trabalho nos CAPS, concomitante à pesquisa. O formulário digital foi elaborado pelos pesquisadores utilizando a aplicação online do Google. Utilizou-se sete perguntas abertas que abordavam a concepção dos profissionais sobre as ações de cuidado compartilhadas entre os CAPS Adulto e CAPS AD, desde os significados sobre o fenômeno até a organização do cuidado e desafios e potencialidades frente aos casos com comorbidades. O questionário foi respondido de forma anônima e as respostas foram enumeradas de 1 a 10 de acordo com cada participante sendo codificadas e identificadas nos resultados como P1, P2, P3 e assim por diante.

Utilizou-se a técnica da análise de conteúdo na modalidade de Análise temática (ROMANINI M, et al., 2017). Esse método busca significados e núcleos de sentido nos achados e, a partir destes núcleos, 
categoriza os resultados. Na primeira fase, organiza-se o material com as respostas dos participantes por leitura flutuante que permite escolher o que será analisado, formular pressupostos e objetivos. Em seguida, classifica-se os temas em categorias analíticas que se relacionam e por fim, faz-se interpretações e inferências sobre o conteúdo dos dados.

Para embasar a discussão utilizou-se outros estudos sobre a temática que fazem parte da literatura científica (MINAYO MC e MARQUES DB, 2008). Este trabalho atende às especificações da Resolução 466/2012, e foi aprovado pelo Comitê de Ética da Escola de Enfermagem da Universidade de São Paulo (EEUSP) sob parecer ํㅜㄴ.981.346/2018.

\section{RESULTADOS}

Com base na análise dos dados emergiram três categorias temáticas: 1) o problema de saúde mental e o uso de álcool e outras drogas, 2) o Projeto Terapêutico Singular (PTS) de casos com duplo diagnóstico e 3) dificuldades e potencialidades no compartilhamento de casos entre CAPS Adulto e CAPS AD.

\section{O problema de saúde mental e o uso de álcool e outras drogas}

Como ilustrado pelas respostas dos profissionais dos CAPS Adulto e CAPS AD, estes consideram que o uso de álcool e outras drogas por pessoas com transtornos mentais é algo cotidiano nas demandas dos serviços. Constatamos que alguns abordam de maneira empírica a percepção de piora nos comportamentos e organização psíquica desses sujeitos quando fazem uso de substâncias, enfatizando uma relação causal entre consumo e desorganização, desagregação, disfunção.

"Temos diversos usuários com essa característica [problemas de saúde mental e de álcool e outras drogas] e entendo que uma crise deve provocar cuidados específicos em cada situação" (P1).

"O uso de drogas está bastante presente e interfere muito, pois induz a ocorrência de crises psiquiátricas aumentando a desorganização do pensamento e destrói o equilíbrio familiar, provocando discussões e desentendimentos. Isso também altera o ambiente terapêutico e a relação profissional/usuário" (P5).

"O uso de substâncias geralmente traz um surgimento ou agravamento dos sintomas de transtorno mental, desorganização geral nos diversos setores da vida, desagregação e conflitos familiares, comportamento disfuncional, complicações por envolvimento em crimes e em situações de risco" (P2).

Por outro lado, outros profissionais abordam esta concepção de forma bilateral e consideram o fenômeno do uso de álcool e outras drogas em sua complexidade e singularidade, não sempre como o problema central e negativo.

"Não necessariamente o uso de álcool e drogas vai interferir no âmbito da organização psíquica da pessoa com transtorno mental" (P3).

"As interferências entre uso de substâncias psicoativas e transtornos mentais têm resultados muito diferentes de acordo com a experiência de vida de cada um. Quando o uso é associado a vivência de alívio de algum mal-estar ou como ajuda para realizar algo desejado pela consciência, pode passar a ser usado repetidas vezes. Há muitas pessoas que apenas fazem uso esporádico, eventual ou recreativo e não tem problemas maiores no uso" (P10).

"Dependendo da frequência ou intensidade, pode interferir na organização psíquica e nas relações em geral" (P4).

\section{O Projeto Terapêutico Singular (PTS) de casos com duplo diagnóstico}

Esta categoria elucida que, para alguns profissionais, a organização das ações de cuidado compartilhadas entre os CAPS depende da relação que o usuário tem com o uso de substâncias e de como este uso agrava os sintomas dos transtornos mentais. Para outros, não difere dos demais. 
"Muitas vezes o uso de drogas é tratado de maneira corriqueira e desconsiderado ou menosprezado. Apenas nos casos mais graves, onde o uso de substâncias impede o relacionamento no serviço ou provoca agressões, é que o usuário é referenciado ao serviço específico" (P8).

"Não diferente dos demais. Entendo que a questão do uso de substâncias psicoativas é mais um elemento a se cuidar, se for algo que gera prejuízo para o sujeito" (P3).

No que tange a construção do PTS, muitas vezes sugerem que intensificar as ações e aumentar a proximidade do usuário com a equipe do CAPS de referência pode não ser suficiente. Todos os profissionais concordam que a construção do PTS para casos com duplo diagnóstico exige uma interlocução dos diferentes serviços da rede, seja para partilhar a discussão dos casos ou para o compartilhamento direto do cuidado.

"É preciso pensar individualmente cada projeto, se faz sentido ser no CAPS AD ou Adulto, ou em ambos ou pensar outros projetos que façam sentido para o sujeito" (P9).

"Em geral necessitam de uma frequência maior ao serviço, de um contato maior com a família e muitas vezes de mais de um serviço de referência, o que implica em discussão de caso com outras equipes" (P7).

"Eventualmente um cuidado compartilhado com outras unidades especializadas de saúde [outros CAPS] ou de atenção social/cultural faz-se necessário. Nem sempre somente a equipe consegue atender integralmente" (P6).

Apenas dois profissionais citaram ações específicas que consideram essenciais para compor o PTS compartilhado desses casos: o acolhimento, o vínculo e a relação terapêutica.

"A abordagem do paciente que tem transtorno mental e tem dependência psicoativas associadas é de tal importância o acolhimento" (P7).

"O cuidado para esses casos é determinado pela relação terapeuta-paciente, visando o estabelecimento do vínculo através de uma boa acolhida, avaliação adequada e estabelecimento de um processo terapêutico pertinente às necessidades daquela pessoa" (P3).

\section{Dificuldades e potencialidades no compartilhamento de casos entre CAPS Adulto e CAPS AD}

Ao ser questionado sobre as ações inerentes ao PTS, é possível identificar no conteúdo das respostas que os profissionais compreendem como potência a possibilidade de compartilhar os casos com diagnóstico de problemas de saúde mental e de uso de álcool e outras drogas. Entendem que a abordagem, os recursos terapêuticos e os planejamentos das ações de cuidado se ampliam e é possível a oferta de um cuidado mais ampliado e integral.

"Entre as potencialidades encontra-se o fato de através dos diversos olhares conseguir orientá-lo em relação ao uso e suas questões de sofrimento psíquico" (P2).

"As potencialidades estão relacionadas com as múltiplas abordagens que podem ser compartilhadas" (P3).

Como dificuldade, identificaram que dar seguimento aos casos compartilhados já é em si um desafio, pois as demandas a serem discutidas geralmente são maiores que a disponibilidade das equipes em estarem juntas para discussão dos casos e alinhamento das ações. A dificuldade na comunicação entre os serviços pode fazer com que as ações de cuidado se sobreponham, criando divergências nas estratégias terapêuticas e dificuldade do usuário em se engajar no PTS proposto em mais de um serviço e com isso, haver negligência de necessidades importantes para aquele sujeito.

"As dificuldades seriam ambas equipes conseguirem manter o contato e a linha de cuidado estabelecida devido à grande demanda e baixa oferta" (P2). 
"Quando há duas equipes juntas, o desafio é manter a coerência do cuidado" (P4).

"Os serviços pouco se conversam e quando fazem parecem tentar dispensar o usuário para outro serviço e não trabalhar de forma conjunta" (P10).

Os participantes consideram que o ideal seria o compartilhamento do cuidado dos usuários com essas características, mas na prática, raras são as vezes que as equipes entendem ser necessário que o cuidado seja compartilhado. Também consideram que casos mais graves tendem a ser mais amplamente discutidos, mas em casos menos complexos a troca de informações e compartilhamento do cuidado fica deficitário.

Apontam que deve haver maior empenho para que esta construção seja coletiva. Fato que evidencia uma possível dificuldade, mas também se entende como uma possibilidade de ampliação e qualificação das estratégias de cuidado.

\section{DISCUSSÃO}

Neste estudo, descrevemos e analisamos as concepções e sentidos dos profissionais sobre as ações de cuidado compartilhadas entre os CAPS Adulto e CAPS AD para usuários com duplo diagnóstico e os desafios e potencialidades na construção de PTS entre as equipes, o que se mostra potente para suscitar discussões e reflexões acerca do tema estudado.

Estudos já comprovaram a relação entre os problemas relacionados ao uso de álcool e outras drogas e a gravidade dos sintomas de saúde mental. Afirmam que as causas da ocorrência da comorbidade em ambos os casos é heterogênea e ainda inconclusiva (CLARO HG, et al., 2015; LUCCHESE R, et al., 2017; CASTILLO-CARNIGLIA A, et al., 2017).

O que se sabe é que a associação causal é recíproca, ou seja, o sofrimento psíquico influência nos problemas relacionados ao uso de substâncias, mas o inverso também é observado. Desta forma, é imprescindível considerar as características da população, a história de vida e psicopatológica e os fatores ambientais, para determinar qual o diagnóstico primário que representa de fato o principal problema para aquela pessoa, assim, o cuidado integral para todas as necessidades poderá ser melhor elaborado (CARRADI-WEBSTER CM, et al., 2020; KRAWCZYK N, et al., 2017).

As respostas de alguns profissionais ilustram suas concepções sobre possíveis consequências do uso de álcool e outras drogas na saúde mental dos usuários, mas não consideram a influência que as questões de saúde mental podem ter no consumo de substâncias. De maneira geral, responsabilizam o uso de álcool e outras drogas pelo agravamento dos sintomas de saúde mental, o que pode dificultar a construção conjunta do PTS e a produção de cuidado, como referiram na categoria 3.

Esta questão é frequentemente mencionada pelas pesquisas e reflete a diferença da construção socioantropológica sobre o usuário de substâncias psicoativas e a pessoa com problemas de saúde mental. Os comportamentos dos indivíduos em que há uma ruptura com a ordem, impõem às equipes de saúde dos serviços territoriais impactos e tensões na medida em que as expectativas da sociedade sobre essa população são pautadas pelo controle e, consequente, modelo disciplinador e estigmatizante, que se contrapõem com a dimensão do cuidado que busca promover a cidadania, a dignidade, a liberdade e a autonomia (VIEIRA FD, et al., 2017).

O preconceito frente à pessoa que faz uso de álcool e outras drogas é constante nas falas e nas atitudes de profissionais de serviços de saúde mental. Esses, por vezes, desconsideram que este seja um problema a ser cuidado em serviços de saúde, como também, atrelam todo e qualquer comportamento desafiador, marginalizado, violento, à moralidade ausente nos usuários de substâncias (CARRADI-WEBSTER CM, et al., 2020; ALVES D, et al., 2018). Além disso, em contextos de criminalização como o do Brasil, pessoas que fazem uso de drogas ilícitas sofrem esses efeitos de forma intensificada (JONES CM e MACCANCE-KATZ $E F, 2019)$.

Ao mesmo tempo, pessoas com problemas de saúde mental tem dificuldades em se engajar em tratamentos para o uso problemático de álcool e outras drogas e referem sofrer discriminação nesses 
cenários. Uma das hipóteses é de que falta esforços das equipes para integrar o cuidado em saúde mental no cuidado ao uso de substâncias (KRAWCZYK N, et al., 2017).

Essa perspectiva está em consonância com um estudo que evidenciou a necessidade dos trabalhadores de saúde mental em estar capacitados para oferecer um cuidado às diferentes necessidades para além da especificidade do serviço em que atua, ou seja, o profissional do CAPS adulto deve também saber manejar situações referentes ao uso problemático de álcool e outras drogas, e os profissionais do CAPS AD, questões intrínsecas aos transtornos mentais e não se limitar ao encaminhamento indiscriminado (PINHO ES, et al., 2018).

Como afirmaram os profissionais na categoria 2 sobre os desafios para a construção do PTS, a complexidade no cuidado desses usuários demanda a integração de outros serviços da rede e recursos humanos. Neste sentido, é preciso transpor os obstáculos criados com a qualificação e aprimoramento da equipe, criação de espaços de diálogos, discussão e supervisão dos casos (LIMA DK e GUIMARÃES J, 2019 OLIVEIRA CA, et al., 2021). Porém, conforme identificado por um estudo realizado na mesma rede de atenção dos CAPS incluídos nessa pesquisa, fragilidades como déficit de recursos humanos, dificuldades de comunicação e compreensão limitada dos profissionais sobre as estratégias possíveis de serem desenvolvidas pela RAPS local, dificultam as ações compartilhadas (NÓBREGA MP, 2017).

Uma estratégia que pode diminuir estas dificuldades e favorecer a construção de um PTS compartilhado, é a criação de uma cultura de matriciamento entre os serviços especializados (CAPS Adulto e CAPS AD). $O$ Apoio Matricial configura-se por um espaço de discussão entre duas ou mais equipes, no intuito de qualificar as ações e produzir saúde de forma compartilhada e integrada. Ele se dá com a participação de uma equipe especializada que propõe intervenções de suporte terapêutico pedagógico, além de poder participar ativamente dos processos de cuidado por meio de atendimentos compartilhados (LIMA M e DIMENSTEIN M, 2016).

A compreensão de que não existe um único modo de fazer o acompanhamento dos usuários diagnosticados com transtornos mental e uso de álcool e outras drogas é unânime entre os profissionais participantes. Enfatizam também que, as características dos serviços especializados devem favorecer minimamente $o$ acolhimento das demandas e das necessidades singulares. Entendendo que são os recursos que devem se adequar às demandas dos sujeitos e não o sujeito se adequar ao serviço (ROMANINI M, et al., 2017).

Como limitações deste estudo, tem-se a ausência de dados sobre a caracterização dos profissionais, o que poderia contribuir com a discussão dos achados; a coleta de dados ter sido realizada com questionário por recurso digital, o que limitou a captação de informações mais abrangentes pelas respostas faladas; e por fim, o número pequeno de participantes e a impossibilidade de generalização dos dados por se tratar de estudo regional tendo como cenário dois CAPS de um mesmo território.

\section{CONCLUSÃO}

A análise da concepção dos profissionais dos CAPS Adulto e CAPS AD estudados reforçou os significados negativos do uso de álcool e outras drogas e sua influência na saúde mental. Por outro lado, sendo esses serviços modelos de atenção abrangentes, concordam que as ações de cuidado compartilhadas para atender os usuários com essa especificidade amplia as possibilidades de cuidado trazendo como contribuição científica ações práticas como o recurso do PTS compartilhado, que pode aprimorar respostas mais integrais, desde que, tenham maior esforço na construção coletiva. Sugere-se novos estudos com foco nos componentes do PTS para ações compartilhadas entre os serviços, na perspectiva de subsidiar práticas baseadas em evidências.

\section{REFERÊNCIAS}

1. ALVES D, et al. A relação entre transtornos mentais e o uso de substâncias psicoativas. Rev Ciência Cena [Internet], $2018 ; 6(1): 38-50$. 
2. ARAÚJO AK, SOARES VL. Trabalho e saúde mental: relato de experiência em um Caps AD III na cidade de João Pessoa, PB. Saúde Debate [Internet], 2018; ;42(spe4):275-84.

3. BOSKA GA, et al. Night beds in psychosocial attention care centers for alcohol and drugs: analysis and characterization. Rev Bras Enferm., 2018; 71(N Esp): 2251-57.

4. BRASIL. Ministério da Saúde. Lei no 10.216, de 6 de abril de 2001. Dispõe sobre a proteção e os direitos das pessoas portadoras de transtornos mentais e redireciona o modelo assistencial em saúde mental. Diário Oficial da União. 2001; 1; 116. Disponível em: http://www.planalto.gov.br/ccivil_03/leis/leis_2001/10216.htm. Acesso em: 26 de março de 2019.

5. BRASIL. Ministério da Saúde. Portaria n 3.088 de 23 de dezembro de 2011. Institui a Rede Atenção Psicossocial para pessoas com sofrimento Mental e com necessidades decorrentes do uso do crack, álcool e outras drogas no âmbito do Sistema Único Saúde (SUS). Diário Oficial da União. 2011; 1; 230. Disponível em: https://bvsms.saude.gov.br/bvs/saudelegis/gm/2011/prt3088_23_12_2011_rep.html. Acesso em: 26 de março de 2019.

6. BRASIL. Ministério da Saúde. Portaria n 336, de 19 de fevereiro de 2002. Dispõe sobre os Centros de Atenção Psicossocial - CAPS, para atendimento público em saúde mental, isto é, pacientes com transtornos mentais severos e persistentes em sua área territorial, em regime de tratamento intensivo, semi-intensivo e não-intensivo. Diário Oficial $\begin{array}{lllll}\text { da } & \text { União. } & 2002 ; & 1 ; & \text { Disponível }\end{array}$ https://bvsms.saude.gov.br/bvs/saudelegis/gm/2002/prt0336_19_02_2002.html. Acesso em: 12 de abril de 2019.

7. CASTILLO-CARNIGLIA A, et al. Psychiatric comorbidities in alcohol use disorder. Lancet Psychiatry, 2019; 6(12): 1068-80.

8. CLARO HG, et al. Drug use, mental health and problems related to crime and violence: Cross-sectional study. Rev Lat Am Enfermagem, 2015; 23(6): 1173-80.

9. CORRADI-WEBSTER CM, et al. Consumo de Drogas, Rede e Apoio Social entre Pacientes Psiquiátricos Ambulatoriais. Aval psicol. [Internet], 2020; 19(2): 132-41.

10. DUARTE MVG, et al. Uso de drogas e cuidado ofertado na Raps: o que pensa quem usa? Saúde Debate. 2020; 127(44):1145-57.

11. FERNANDES MA, et al. Transtornos mentais e comportamentais por uso de substâncias psicoativas em hospital psiquiátrico. SMAD Rev Eletrônica Saúde Ment Álcool e Drog., 2018; 13(2): 64-70.

12. HUNT GE, et al. Prevalence of comorbid substance use in schizophrenia spectrum disorders in community and clinical settings, 1990-2017: Systematic review and meta-analysis. Drug Alcohol Depend., 2018;191(Epub): 234-58.

13. JONES CM, MCCANCE-KATZ EF. Co-occurring substance use and mental disorders among adults with opioid use disorder. Drug Alcohol Depend. 2019; 197(Epub):78-82.

14. KRAWCZYK N, et al. The association of psychiatric comorbidity with treatment completion among clients admitted to substance use treatment programs in a U.S. national sample. Drug Alcohol Depend., 2017; 175(Epub): $157-63$.

15. LIMA DK, GUIMARÃES J. A Rede de Atenção Psicossocial sob o olhar da complexidade: quem cuida da saúde mental? Saúde Debate. 2019; 122(43): 883-96.

16. LIMA M, DIMENSTEIN M. O apoio matricial em saúde mental: Uma ferramenta apoiadora da atenção à crise. Interface Commun Heal Educ., 2016; 20(58): 625-35.

17. LUCCHESE R, et al. Transtorno mental comum entre indivíduos que abusam de álcool e drogas: Estudo transversal. Texto e Context Enferm., 2017; 26(1): e4480015.

18. MESSER T, et al. Substance abuse in patients with bipolar disorder: A systematic review and meta-analysis. Psychiatry Research, 2017; 253: 338-50.

19. MINAYO MC. O desafio do conhecimento. Pesquisa qualitativa em saúde. 11ª ed. São Paulo: Hucitec, 2008.

20. NÓBREGA MP, et al. Tecendo a rede de atenção psicossocial oeste do município de São Paulo. Rev Bras Enferm. [Internet]; 2017; 70(5): 1016-23.

21. OLIVEIRA CA, et al. Projeto terapêutico singular (PTS): instrumento de cuidado ao sujeito em sofrimento psíquico. Revista Eletrônica Acervo Saúde, 2021; 13(2): e5709.

22. PINHO ES, et al. Working processes of professionals at Psychosocial Care Centers (CAPS): an integrative review. Ciênc. saude coletiva, 2018; 23(1): 141-52.

23. ROMANINI M, et al. O conceito de acolhimento em ato: reflexões a partir dos encontros com usuários e profissionais da rede. Saúde Debate [Internet]. 2017; 41(113): 486-99.

24. TREVISAN ER, CASTRO SS. Centros de Atenção Psicossocial - álcool e drogas: perfil dos usuários. Saúde Debate, 2019; 121(43):450-63.

25. URQUIZA MD, MARQUES DB. Análise de conteúdo em termos de Bardin aplicada à comunicação corporativa sob o signo de uma abordagem teórico-empírica. Entretextos, 2016; 16(1): 115-44

26. VIEIRA FD, et al. Consumo de drogas por pessoas com diagnósticos psiquiátricos: Percursos possíveis em uma rede de atenção psicossocial. Physis, 2017; 27(4): 1243-63. 